Cahiers $d u$ MONDE RUSSE

\section{Cahiers du monde russe}

Russie - Empire russe - Union soviétique et États indépendants

$62 / 4 \mid 2021$

Varia

\title{
ИСТОРИЯ СОВЕТСКОЙ МЕДИЦИНЫ. ОБЗОР НОВЕЙШИХ ИССЛЕДОВАНИЙ (2010-2020 ГГ.)
}

[History of Soviet Medicine: A Review of the Newest Studies (2010-2020)]

\section{Владислав Яковенко}

\section{OpenEdition}

\section{Journals}

Édition électronique

URL : https://journals.openedition.org/monderusse/13019

DOI : 10.4000/monderusse. 13019

ISSN : $1777-5388$

\section{Éditeur}

Éditions de l'EHESS

\section{Édition imprimée}

Date de publication : 1 décembre 2021

Pagination : 815-828

ISBN : 978-2-7132-2895-7

ISSN : $1252-6576$

\section{Référence électronique}

Владислав Яковенко, «История советской медицины. Обзор новейших исследований (20102020 гг.)», Cahiers du monde russe [Онлайн], 62/4 | 2021, Выложить онлайн 01 décembre 2021, Наводить справки в 03 septembre 2022. URL: http://journals.openedition.org/monderusse/13019 ; DOI: https://doi.org/10.4000/monderusse. 13019

Ce document a été généré automatiquement le 3 septembre 2022.

All rights reserved 


\section{ИСТОРИЯ СОВЕТСКОЙ МЕДИЦИНЫ. ОБЗОР НОВЕЙШИХ ИССЛЕДОВАНИЙ (2010-2020 Гг.)}

[History of Soviet Medicine: A Review of the Newest Studies (2010-2020)]

\section{Владислав Яковенко}

1 В 2011 Г. СПЕЦИАЛИСТ ПО РОССИЙСКОЙ ИСТОРИИ АНДРЕАС РЕННЕР ОТМЕТИЛ, ЧТО ИСТОРИКО-МЕДИЦИНСКИЕ ИССЛЕДОВАНИЯ В МАССЕ СВОЕЙ ЕВРОПОЦЕНТРИЧНЫ, А РОССИЯ В НИХ ЗА ИСКЛЮЧЕНИЕМ НОВАТОРСКИХ РАБОТ НЭНСИ ФРИДЕН, ДЖОНА АЛЕКСАНДЕРА, ДЖОНА ХАТЧИНСОНА И СЮЗАН ГРОСС СОЛОМОН ИЗУЧАЕТСЯ В ОСНОВНОМ В ИСТОРИКО-БИОГРАФИЧЕСКОМ И ИСТОРИКО-ДИСЦИПЛИНАРНОМ КЛЮЧЕ, СВОЙСТВЕННОМ ТРАДИЦИОННОЙ ИСТОРИИ МЕДИЦИНЫ ${ }^{1}$. ВМЕСТЕ С ТЕМ ОН УКАЗАЛ НА НАЧАВШЕЕСЯ РАСШИРЕНИЕ СФЕРЫ ИССЛЕДОВАНИЙ В ЭТОЙ ОБЛАСТИ ${ }^{2}$. ЦЕЛЬ МОЕГО ОБЗОРА - ПРОДЕМОНСТРИРОВАТЬ В КАКИХ НАПРАВЛЕНИЯХ ПРОИСХОДИТ УКАЗАННОЕ РАСШИРЕНИЕ. Я ОБРАЩАЮСЬ К ИСТОРИОГРАФИИ СОВЕТСКОЙ МЕДИЦИНЫ, ЧТОБЫ РАССМОТРЕТЬ ОСНОВНЫЕ ВЕКТОРЫ, СУЩЕСТВУЮЩИЕ В ЭТОМ ИССЛЕДОВАТЕЛЬСКОМ ПОЛЕ. В ОТЛИЧИЕ ОТ НЕКОТОРЫХ СУЩЕСТВУЮЩИХ ОБЗОРНЫХ СТАТЕЙ, МОЯ РАБОТА СКОНЦЕНТРИРОВАНА НА СОВРЕМЕННОМ СОСТОЯНИИ ИСТОРИОГРАФИИ ${ }^{3}$. ПОЭТОМУ В ТЕКСТЕ УЧТЕНЫ ТОЛЬКО ПУБЛИКАЦИИ, ВЫШЕДШИЕ ИЗ ПЕЧАТИ ЗА ПЕРИОД С 2010 ПО 2020 ГГ., ЧТО ПОЗВОЛЯЕТ ОХВАТИТЬ ДОСТАТОЧНЫЙ ПЛАСТ ЛИТЕРАТУРЫ. ПОСКОЛЬКУ Я СТАРАЮСЬ ПОКАЗАТЬ РАЗНООБРАЗИЕ СУЩЕСТВУЮЩИХ ИССЛЕДОВАТЕЛЬСКИХ НАПРАВЛЕНИЙ, Я НЕ ОГРАНИЧИВАЮСЬ ОТДЕЛЬНЫМИ ТЕМАМИ, ИНТЕРЕСУЮЩИМИ ИСТОРИКОВ СОВЕТСКОЙ МЕДИЦИНЫ, И НЕ ФОКУСИРУЮСЬ ИСКЛЮЧИТЕЛЬНО НА КНИГАХ, КАК ЭТО ДЕЛАЮТ АВТОРЫ НЕКОТОРЫХ ОБЗОРОВ ${ }^{4}$. 


\section{История институтов советского здравоохранения}

2 КАК И РАНЕЕ, ИСТОРИКИ СОВЕТСКОЙ МЕДИЦИНЫ УДЕЛЯЮТ ВНИМАНИЕ ФОРМИРОВАНИЮ И РАБОТЕ МЕДИЦИНСКИХ СЛУЖБ И УЧРЕЖДЕНИЙ: НАУЧНОИССЛЕДОВАТЕЛЬСКИХ ИНСТИТУТОВ, БОЛЬНИЦ, ГОСПИТАЛЕЙ И ДРУГИХ ОРГАНИЗАЦИЙ. С ОДНОЙ СТОРОНЫ, УЧЕНЫЕ РАБОТАЮТ С ИСТОРИЕЙ ВСЕСОЮЗНЫХ СТРУКТУР. ИСТОРИЯ СОЗДАНИЯ ТАКОЙ КРУПНОЙ ОРГАНИЗАЦИИ КАК АКАДЕМИЯ МЕДИЦИНСКИХ НАУК СССР ПОДРОБНО ИЗУЧЕНА В СТАТЬЕ СЕРГЕЯ ГЛЯНЦЕВА И АННЫ СТОЧИК. АВТОРЫ ПОКАЗАЛИ, ЧТО ПОЯВЛЕНИЕ ЭТОЙ МАСШТАБНОЙ СТРУКТУРЫ ПРОИЗОШЛО ИЗ-ЗА РОСТА КОЛИЧЕСТВА И КАЧЕСТВА МЕДИЦИНСКИХ ИССЛЕДОВАНИЙ, ЖЕЛАНИЯ УСТАНОВИТЬ ЕДИНУЮ УПРАВЛЕНЧЕСКУЮ ВЕРТИКАЛЬ В ЭТИХ ИССЛЕДОВАНИЯХ, СОЕДИНИТЬ В НЕЙ НАУКИ О ЗДОРОВЫХ И БОЛЬНЫХ ЛЮДЯХ, А ТАКЖЕ ТЕОРИЮ И ПРАКТИКУ МЕДИЦИНЫ. ВСЕ ЭТО, СЧИТАЮТ АВТОРЫ, ДЕЛАЛОСЬ С ЦЕЛЬЮ ВЫВЕСТИ СОВЕТСКУЮ МЕДИЦИНУ НА МИРОВОЙ УРОВЕНЬ 5 .

З С ДРУГОЙ СТОРОНЫ, В ИСТОРИОГРАФИИ ВСТРЕЧАЮТСЯ ИССЛЕДОВАНИЯ О МЕНЕЕ КРУПНЫХ СТРУКТУРАХ, ВАЖНЫХ В ОПРЕДЕЛЕННОЙ СФЕРЕ МЕДИЦИНЫ И ДЛЯ ОТДЕЛЬНЫХ ТЕРРИТОРИЙ. ТАК, В СТАТЬЕ МАРИНЫ СВИНЦОВОЙ НА ОСНОВЕ АРХИВНЫХ И ОПУБЛИКОВАННЫХ ИСТОЧНИКОВ РЕКОНСТРУИРОВАНА ДЕЯТЕЛЬНОСТЬ КИРОВСКОГО ИНСТИТУТА ЭПИДЕМИОЛОГИИ И МИКРОБИОЛОГИИ В 1941-1945 ГГ. И ПОКАЗАНА ЕГО РОЛЬ В БОРЬБЕ С ЭПИДЕМИЯМИ В КИРОВСКОЙ ОБЛАСТИ, А ТАКЖЕ В ПРОИЗВОДСТВЕ СЫВОРОТОК И ВАКЦИН ДЛЯ НЕСКОЛЬКИХ РЕГИОНОВ СССР 6.

\section{Биографические исследования и изучение научных школ советской медицины}

4 ТРАДИЦИОННЫМ ЖАНРОМ В ИСТОРИИ МЕДИЦИНЫ ЯВЛЯЮТСЯ ИССЛЕДОВАНИЯ СКОНЦЕНТРИРОВАННЫЕ НА КОНКРЕТНЫХ УЧЕНЫХ. ПОДОБНОГО РОДА РАБОТ ДОВОЛЬНО МНОГО: В НЕКОТОРЫХ ПРОФИЛЬНЫХ ИЗДАНИЯХ БИОГРАФИИ ВРАЧЕЙ СОСТАВЛЯЮТ БОЛЕЕ ПОЛОВИНЫ ИСТОРИКО-МЕДИЦИНСКИХ ПУБЛИКАЦИЙ. ТАКИЕ ИССЛЕДОВАНИЯ СКОНЦЕНТРИРОВАНЫ НА СКРУПУЛЕЗНОМ СБОРЕ ФАКТОВ О НАУЧНОЙ И ОРГАНИЗАТОРСКОЙ РАБОТЕ ОТДЕЛЬНЫХ ВРАЧЕЙ. ПОСКОЛЬКУ НАИБОЛЕЕ КРУПНЫЕ ФИГУРЫ СОВЕТСКОЙ МЕДИЦИНЫ ИССЛЕДОВАНЫ ОЧЕНЬ ПОДРОБНО, ИСТОРИКИ ЗАЧАСТУЮ ОБРАЩАЮТСЯ К БИОГРАФИЯМ ЧУТЬ МЕНЕЕ ИЗВЕСТНЫХ СПЕЦИАЛИСТОВ ${ }^{7}$ ВМЕСТЕ С ТЕМ СОВЕТСКИЕ БИОГРАФИИ ВЕДУЩИХ МЕДИКОВ ПРИЗНАЮТСЯ СОВРЕМЕННЫМИ УЧЕНЫМИ ИДЕОЛОГИЗИРОВАННЫМИ, А ПОТОМУ НАБЛЮДАЮТСЯ ПОПЫТКИ ИЗУЧИТЬ ПРОФЕССИОНАЛЬНЫЙ ПУТЬ ЭТИХ ЛЮДЕЙ, НЕ НАХОДЯСЬ ПОД ДАВЛЕНИЕМ ОФИЦИАЛЬНОГО СОВЕТСКОГО НАРРАТИВА ${ }^{8}$.

НАРЯДУ С БИОГРАФИЯМИ ПЕРВОГО ТИПА ВЫХОДЯТ И ДРУГИЕ ИССЛЕДОВАНИЯ В ЭТОМ ЖАНРЕ. ИХ АВТОРЫ СТРЕМЯТСЯ РАССМОТРЕТЬ РАЗЛИЧНЫЕ СТОРОНЫ ЖИЗНИ МЕДИКОВ В ИХ ВЗАИМОСВЯЗИ, НЕ ОГРАНИЧИВАЯСЬ ПРОФЕССИОНАЛЬНОЙ ДЕЯТЕЛЬНОСТЬЮ. ЯРКИМ ПРИМЕРОМ ТАКОГО ИССЛЕДОВАНИЯ СЛУЖИТ КНИГА НИКОЛАЯ КРЕМЕНЦОВА «Martian Stranded on Earth: Alexander Bogdanov, Blood Transfusions, and Proletarian Science», В КОТОРОЙ ИСТОРИК РАССМАТРИВАЕТ ПИСАТЕЛЬСКУЮ, МЕДИЦИНСКУЮ И ПОЛИТИЧЕСКУЮ СТОРОНЫ ЖИЗНИ ИЗВЕСТНОГО «СТАРОГО БОЛЬШЕВИКА» АЛЕКСАНДРА БОГДАНОВА $С$ УЧЕТОМ ИХ ВЗАИМОПРОНИКНОВЕНИЯ. МОЖНО 
СПРАВЕДЛИВО ЗАМЕТИТЬ, ЧТО ПОДОБНОЕ ДЕЛАЛОСЬ И РАНЕЕ. НО ИМЕННО КРЕМЕНЦОВ УДАЧНО ПОКАЗАЛ, ЧТО БОГДАНОВСКИЙ КОНЦЕПТ ПРОЛЕТАРСКОЙ НАУКИ, ВПЕРВЫЕ ВЫСКАЗАННЫЙ ИМ В НАУЧНОЙ ФАНТАСТИКЕ, СИЛЬНО ПОВЛИЯЛ НА ДАЛЬНЕЙШУЮ НАУЧНУЮ ПОЛИТИКУ В СОВЕТСКОЙ РОССИИ. КАК ОТМЕЧАЕТ АВТОР, ПАРТИЙНЫЕ СВЯЗИ ПОЗВОЛИЛИ БОГДАНОВУ СОЗДАТЬ БОЛЬШОЕ ИССЛЕДОВАТЕЛЬСКОЕ УЧРЕЖДЕНИЕ, НО ЕГО УПРАВЛЕНЧЕСКИЙ СТИЛЬ И РЕАЛЬНЫЕ НАУЧНЫЕ ПРАКТИКИ БЫЛИ АКТУАЛЬНЫ СКОРЕЕ ДЛЯ ХІХ В., ЧЕМ ДЛЯ СОВЕТСКОЙ НАУКИ. К ЭТОЙ ЖЕ КАТЕГОРИИ МОЖНО ОТНЕСТИ КНИГУ ДЭНИЕЛА ТОДЕСА «Ivan Pavlov: A Russian Life in Science». ИСТОРИК АНАЛИЗИРУЕТ НЕ ТОЛЬКО НАУЧНЫЙ ПУТЬ ИЗВЕСТНОГО ФИЗИОЛОГА, НО ТАКЖЕ НЕПРОСТЫЕ ОТНОШЕНИЯ УЧЕНОГО С СОВЕТСКИМ ПОЛИТИЧЕСКИМ РУКОВОДСТВОМ И ЕГО ЛИЧНОСТНЫЕ КАЧЕСТВА В ТЕСНОЙ ВЗАИМОСВЯЗИ ${ }^{10}$.

6 К БИОГРАФИЧЕСКИМ ПУБЛИКАЦИЯМ ПРИЛЕГАЮТ ИССЛЕДОВАНИЯ НАУЧНЫХ ШКОЛ СОВЕТСКОЙ МЕДИЦИНЫ. ЗАЧАСТУЮ ТАКИЕ МАТЕРИАЛЫ ТОЖЕ ВЫСТРОЕНЫ ВОКРУГ ОДНОГО ЧЕЛОВЕКА - ОСНОВАТЕЛЯ ТОГО ИЛИ ИНОГО НАУЧНОГО НАПРАВЛЕНИЯ. НАПРИМЕР, В СТАТЬЕ ВЛАДИМИРА БОРОДУЛИНА И АЛЕКСЕЯ ТОПОЛЯНСКОГО ПРОСЛЕЖИВАЕТСЯ ФОРМИРОВАНИЕ ГАСТРОЭНТЕРОЛОГИИ И ДИЕТОЛОГИИ В СССР ВОКРУГ ИЗВЕСТНОГО СОВЕТСКОГО ВРАЧА М.И. ПЕВЗНЕРА ${ }^{11}$. С ТОЙ ЖЕ ТОЧКИ ЗРЕНИЯ РОМАН ФАНДО АНАЛИЗИРУЕТ ШКОЛУ МЕДИЦИНСКОЙ ГЕНЕТИКИ ПОД РУКОВОДСТВОМ С.Г. ЛЕВИТА В МОСКВЕ ${ }^{12}$.

\section{Врачи и политики в СССР}

7 ПОЛИТИЧЕСКИЙ КОНТЕКСТ МЕДИЦИНЫ ВОЗНИКАЕТ НЕ ТОЛЬКО В БИОГРАФИЧЕСКИХ ИССЛЕДОВАНИЯХ О ДОКТОРАХ. ВНИМАНИЕ ИСТОРИКОВ ПРИВЛЕКАЕТ ПРОБЛЕМА ОТНОШЕНИЙ СОВЕТСКОЙ ВЛАСТИ С ВРАЧАМИ КАК С ПРОФЕССИОНАЛЬНОЙ КОРПОРАЦИЕЙ, ОСОБЕННО В ПЕРВЫЕ ГОДЫ СОВЕТСКОЙ ВЛАСТИ. ТАК, ИРИНА ЕГОРЫШЕВА ОБРАЩАЕТ ВНИМАНИЕ НА МАССОВЫЕ АРЕСТЫ ДОКТОРОВ В ЭТОТ ПЕРИОД И НА РАБОТУ НАРОДНОГО КОМИССАРА ЗДРАВООХРАНЕНИЯ Н.А.СЕМАШКО ПО ИХ ОСВОБОЖДЕНИЮ ${ }^{13}$. ЗДЕСЬ СЕМАШКО ВМЕСТЕ С КОЛЛЕГАМИ ПО ЦЕХУ ПРОТИВОПОСТАВЛЕНЫ РЕПРЕССИВНОЙ МАШИНЕ И В ЧАСТНОСТИ ВЧК. ОБРАТНУЮ ПОЗИЦИЮ ЗАНИМАЕТ ВИКТОР ТОПОЛЯНСКИЙ, КОТОРЫЙ ПИШЕТ ОБ УЧАСТИИ НАРКОМА В РЕПРЕССИЯХ ПРОТИВ ВРАЧЕЙ, СТОЯВШИХ В ОППОЗИЦИИ К БОЛЬШЕВИКАМ ${ }^{14}$. И В ЭТОМ СЛУЧАЕ НАРКОМ ЗДРАВООХРАНЕНИЯ РАССМАТРИВАЕТСЯ УЖЕ В КАЧЕСТВЕ ПАРТИЙНОГО И ГОСУДАРСТВЕННОГО ФУНКЦИОНЕРА, КОТОРЫЙ ИСПОЛЬЗУЕТ РЕПРЕССИИ В СВОИХ ИНТЕРЕСАХ, И ПРОТИВОПОСТАВЛЯЕТСЯ МЕДИЦИНСКОМУ СООБЩЕСТВУ.

СМЕЖНЫЕ ВОПРОСЫ ПРИМЕНИТЕЛЬНО К ПЕРИОДУ 1930-Х ГГ. ИССЛЕДУЕТ ИГОРЬ ПОЛЯНСКИЙ. ОН АНАЛИЗИРУЕТ, КАК МЕДИЦИНСКАЯ ДИСКУССИЯ ПО ПОВОДУ ИСКУССТВЕННОГО ПНЕВМОТОРАКСА В ЛЕЧЕНИИ ТУБЕРКУЛЕЗА ПЕРЕШЛА В ПОЛИТИЧЕСКОЕ ПРОСТРАНСТВО И ЗАКОНЧИЛАСЬ РАССТРЕЛОМ ОДНОГО ИЗ УЧАСТНИКОВ СПОРА ${ }^{15}$. ВМЕСТЕ С ТЕМ, КАК ПОКАЗЫВАЕТ НА ПРИМЕРЕ ЛЕНИНГРАДА КРИСТОФЕР УИЛЬЯМС, НЕ ТОЛЬКО ОТДЕЛЬНЫЕ СПЕЦИАЛИСТЫ, НО И МЕДИКИ КАК ПРОФЕССИОНАЛЬНАЯ ГРУППА ЗНАЧИЛИСЬ В СТАЛИНСКОМ СПИСКЕ НЕБЛАГОНАДЕЖНЫХ. СОГЛАСНО ИСТОРИКУ, ИМЕННО НА НИХ В 1937-1938 ГГ. И.В. СТАЛИН ПЕРЕЛОЖИЛ ВСЮ ОТВЕТСТВЕННОСТЬ ЗА НЕУДАЧИ СОВЕТСКОГО 
ЗДРАВООХРАНЕНИЯ, ПРИЧИНЫ КОТОРЫХ НА САМОМ ДЕЛЕ БЫЛИ ЗНАЧИТЕЛЬНО БОЛЕЕ КОМПЛЕКСНЫМИ ${ }^{16}$.

\section{Советский медицинский дискурс как властный}

КАК ПОКАЗАЛ МИШЕЛЬ ФУКО, МЕДИЦИНСКИЙ ДИСКУРС ЯВЛЯЕТСЯ ОДНИМ ИЗ НАИБОЛЕЕ ВЛАСТНЫХ. СОВРЕМЕННЫЕ СПЕЦИАЛИСТЫ ИЗУЧАЮТ СОВЕТСКУЮ МЕДИЦИНУ В ЭТОМ КЛЮЧЕ. КАК ДОКАЗЫВАЕТ НАТАЛЬЯ ТАМАРУЧИ, В РАМКАХ СОВЕТСКОЙ СИСТЕМЫ ТЕЛО ЧЕЛОВЕКА ПЕРЕСТАВАЛО БЫТЬ ЕГО СОБСТВЕННОСТЬЮ, А СТАТУС ЗДОРОВОГО ИЛИ БОЛЬНОГО ЕМУ ПРИСВАИВАЛИ ГОСУДАРСТВЕННЫЕ СТРУКТУРЫ ${ }^{17}$. НО ЛюДЕЙ РАЗДЕЛЯЛИ НЕ ТОЛЬКО НА БОЛЬНЫХ И ЗДОРОВЫХ. НА ПРИМЕРЕ ПУБЛИЦИСТИКИ Н.А.СЕМАШКО НЕДАВНЕЕ ИССЛЕДОВАНИЕ ДЕМОНСТРИРУЕТ, ЧТО ПОСРЕДСТВОМ МЕДИКО-ПОЛИТИЧЕСКОЙ ГИБРИДИЗАЦИИ СОВЕТСКАЯ МЕДИЦИНА ФОРМИРОВАЛА СТРАТИФИКАЦИЮ НАСЕЛЕНИЯ, ВКЛЮЧАВШУЮ СОЦИАЛЬНЫЕ ГРУППЫ С РАЗЛИЧНЫМИ ХАРАКТЕРИСТИКАМИ И УРОВНЯМИ ДОСТУПА К РЕСУРСАМ $^{18}$. НЕХВАТКА РАЗНООБРАЗНЫХ МЕДИЦИНСКИХ РЕСУРСОВ ОСТРО ОЩУЩАЛАСЬ В СССР, ПО МЕНЬШЕЙ МЕРЕ, ВЕСЬ ДОВОЕННЫЙ ПЕРИОД. КАК УТВЕРЖДАЕТ ТРИША СТАРКС, ОТЧАСТИ ЭТУ ПРОБЛЕМУ БЫЛО ПРИЗВАНО РЕШИТЬ САНИТАРНОЕ ПРОСВЕЩЕНИЕ, ПРОДВИГАВШЕЕ МЕДИЦИНСКУЮ ПРОФИЛАКТИКУ. ИССЛЕДОВАТЕЛЬНИЦА ПРЕДЛАГАЕТ РАСЦЕНИВАТЬ СОВЕТСКИЙ САНПРОСВЕТ В КАЧЕСТВЕ НАБОРА ПОЛИТИЧЕСКИХ ПОСЛАНИЙ, КОТОРЫЕ ПАРАЛЛЕЛЬНО ПОСТУЛИРОВАЛИ ДОСТИЖЕНИЯ СОЦИАЛИСТИЧЕСКОГО ЗДРАВООХРАНЕНИЯ ВНУТРИ СТРАНЫ И ВОВНЕ ${ }^{19}$.

\section{Экспертный статус и полномочия советских врачей}

ВЛАСТНОСТЬ ДИСКУРСА НЕ ВОЗМОЖНА БЕЗ ПРИЗНАНИЯ ЭКСПЕРТНОГО СТАТУСА ВРАЧЕЙ И УТВЕРЖДЕНИЯ ШИРОТЫ ИХ ПОЛНОМОЧИЙ. ЭТОТ ВОПРОС ОСТАЕТСЯ ВАЖНЫМ ДЛЯ ИССЛЕДОВАТЕЛЕЙ СОВЕТСКОЙ МЕДИЦИНЫ НАЧИНАЯ С ХХ В. КАК ПОКАЗЫВАЕТСЯ В СТАТЬЕ ИРИНЫ СИРОТКИНОЙ, СООБЩЕСТВО РОССИЙСКИХ ПСИХИАТРОВ ПОЛУЧИЛО ПОСЛЕ РЕВОЛЮЦИИ ШИРОЧАЙШИЕ ПОЛНОМОЧИЯ И ПОВЫСИЛО СВОЙ СТАТУС В ПРОФЕССИОНАЛЬНОЙ СРЕДЕ, РАСПЛАТИВШИСЬ ЗА ЭТО ПОДДЕРЖКОЙ СОВЕТСКОЙ ЦЕНТРАЛИЗАЦИИ И БЮРОКРАТИЗАЦИИ МЕДИЦИНЫ ${ }^{20}$. С ПОХОЖИМ ТЕЗИСОМ ВЫСТУПАЕТ ГРЕГОРИ

ДЮФО, КОТОРЫЙ АНАЛИЗИРУЕТ СОВЕТСКУЮ ВНЕБОЛЬНИЧНУЮ ПСИХИАТРИЮ В 1920-Х НАЧАЛЕ 1930-Х ГГ. ОН УБЕДИТЕЛЬНО ДОКАЗЫВАЕТ, ЧТО, БЛАГОДАРЯ СОЗВУЧНОСТИ СУЩЕСТВОВАВШИХ ПСИХИАТРИЧЕСКИХ ТЕОРИЙ КУРСУ НОВЫХ ВЛАСТЕЙ НА СОЗДАНИЕ НОВОГО ЧЕЛОВЕКА, ПСИХИАТРЫ ПОЛУЧИЛИ ПОЛНОМОЧИЯ ДЛЯ ВТОРЖЕНИЯ В СОЦИАЛЬНУЮ СФЕРУ 21 . НА СМЕЖНУЮ ТЕМУ ВЫСКАЗЫВАЕТСЯ МИХАИЛ ПОГОРЕЛОВ В СТАТЬЕ О СОВЕТСКОЙ СУДЕБНОЙ ПСИХИАТРИИ. ЧЕРЕЗ ИЗУЧЕНИЕ ПОНЯТИЯ «ПСИХОПАТИЯ» АВТОР ПОКАЗЫВАЕТ, КАК РАСШИРЯЛИСЬ И СУЖАЛИСЬ ГРАНИЦЫ ПРОФЕССИОНАЛЬНОЙ ЮРИСДИКЦИИ СУДЕБНОЙ ПСИХИАТРИИ, КОТОРАЯ ФУНКЦИОНИРОВАЛА ОДНОВРЕМЕННО В СФЕРАХ ЮРИСПРУДЕНЦИИ И МЕДИЦИНЫ ${ }^{22}$. ВТОРИТ ЭТОМУ ИСТОРИК ПАВЕЛ ВАСИЛЬЕВ, РАБОТА КОТОРОГО ХОРОШО ДЕМОНСТРИРУЕТ ШИРОТУ ВЛИЯНИЯ СОВЕТСКИХ ВРАЧЕЙ. ИСПОЛЬЗУЯ ПРИМЕР ПЕТРОГРАДА/ЛЕНИНГРАДА, ОН ОБРАЩАЕТ ВНИМАНИЕ НА ТО, ЧТО, БЛАГОДАРЯ 
ДЕЙСТВИЯМ СОВЕТСКИХ МЕДИКОВ, К НАЧАЛУ 1930-Х ГГ. РЫНОК НАРКОТИКОВ НАХОДИЛСЯ ПОД ЖЕСТКИМ КОНТРОЛЕМ ГОСУДАРСТВА, ТОРГОВЛЯ ПСИХОАКТИВНЫМИ ВЕЩЕСТВАМИ БЫЛА ПРИЗНАНА НЕЗАКОННОЙ, А ВРАЧИ И КРИМИНОЛОГИ СТАЛИ КЛЕЙМИТЬ НАРКОМАНОВ КАК БУРЖУАЗНЫХ ДЕВИАНТОВ ${ }^{23}$. МЕДИЦИНА ТАКЖЕ УМНОЖАЛА СВОЕ ВЛИЯНИЕ ЗА СЧЕТ ПОДКЛЮЧЕНИЯ К АНТИРЕЛИГИОЗНОЙ ПОВЕСТКЕ СОВЕТСКОЙ ВЛАСТИ. КАК СООБЩАЕТ ИГОРЬ ПОЛЯНСКИЙ, В 1920-Х ГГ. РЕЛИГИОЗНОЕ ПОВЕДЕНИЕ ЛЮДЕЙ БЫЛО ПЕРЕКОДИРОВАНО СОВЕТСКИМИ ДОКТОРАМИ В ТЕРМИНАХ БОЛЕЗНЕЙ И СИМПТОМОВ ${ }^{24}$. ОДНАКО, КАК ПОКАЗЫВАЕТ БЕНДЖАМИН ЗАИЦЕК НА ПРИМЕРЕ ПСИХИАТРОВ, УЖЕ В 1930-Х ГГ. СОВЕТСКАЯ ВЛАСТЬ УСТАНОВИЛА ЖЕСТКИЕ ГРАНИЦЫ ПРОФЕССИОНАЛЬНОЙ ЮРИСДИКЦИИ КАК МИНИМУМ ДЛЯ НЕКОТОРЫХ МЕДИЦИНСКИХ СПЕЦИАЛИЗАЦИЙ ${ }^{25}$.

\section{Врачи, фельдшеры и медсестры в Советском Союзе}

ТЕСНО СВЯЗАН С ТЕМОЙ ЭКСПЕРТНОГО СТАТУСА ВРАЧЕЙ В СССР ВОПРОС О ПОЛОЖЕНИИ ОСТАЛЬНОГО МЕДИЦИНСКОГО ПЕРСОНАЛА, ЧАСТЬ ИЗ КОТОРОГО ТАКЖЕ ПРЕТЕНДОВАЛА НА РАСШИРЕНИЕ ПОЛНОМОЧИЙ. СЭМУЭЛ РЭМЕР ОТМЕЧАЕТ, ЧТО ФЕЛЬДШЕРЫ УЖЕ В 1905 Г. СОЗДАЛИ СИЛЬНОЕ ПРОФЕССИОНАЛЬНОЕ ДВИЖЕНИЕ И НАЧАЛИ ДОБИВАТЬСЯ РАВНЫХ С ВРАЧАМИ ПРАВ. ОДНАКО СОВЕТСКАЯ ВЛАСТЬ ПОДТВЕРДИЛА ИСКЛЮЧИТЕЛЬНЫЙ ЭКСПЕРТНЫЙ СТАТУС ВРАЧЕЙ И СНАЧАЛА ДАЖЕ ХОТЕЛА ПОЛНОСТЬЮ ОТКАЗАТЬСЯ ОТ ОБУЧЕНИЯ ФЕЛЬДШЕРОВ. ВПРОЧЕМ, СООБЩАЕТ АВТОР, К 1930-М ГГ. ИХ ПОДГОТОВКА БЫЛА ВОССТАНОВЛЕНА ${ }^{26}$. С ДРУГОЙ СТОРОНЫ, УКАЗЫВАЕТ СЮЗАН ГРАНТ, В ОТЛИЧИЕ ОТ ВРАЧЕЙ И ФЕЛЬДШЕРОВ, ПРОФЕССИОНАЛИЗАЦИЯ СООБЩЕСТВА РОССИЙСКИХ МЕДСЕСТЕР ПРОИЗОШЛА ЛИШЬ В КОНЦЕ 1930-Х ГГ. ПОЭТОМУ ОНИ ДОЛГОЕ ВРЕМЯ БЫЛИ ИСКЛЮЧЕНЫ ИЗ ОБСУЖДЕНИЙ СОБСТВЕННОГО ПРОФЕССИОНАЛЬНОГО ПОЛОЖЕНИЯ ${ }^{27}$.

\section{Было ли (без)успешно советское здравоохранение?}

НЕ МЕНЕЕ ВАЖНЫМ ДЛЯ ИССЛЕДОВАТЕЛЕЙ ЯВЛЯЕТСЯ ВОПРОС О ТОМ, БЫЛО ЛИ ЗДРАВООХРАНЕНИЕ В СССР УСПЕШНЫМ ПРОЕКТОМ. СОВЕТСКАЯ ИСТОРИКОМЕДИЦИНСКАЯ ТРАДИЦИЯ ВО МНОГОМ СТРОИЛАСЬ НА ПОДДЕРЖАНИИ ПРЕДСТАВЛЕНИЯ О ПОСТУПАТЕЛЬНЫХ ПОБЕДАХ ОТЕЧЕСТВЕННЫХ ВРАЧЕЙ ПОСЛЕ ОКТЯБРЯ 1917 Г. В НОВЕЙШИХ ПУБЛИКАЦИЯХ ЭТО МНЕНИЕ БЫЛО ПОСТАВЛЕНО ПОД ВОПРОС. ПРИМЕРОМ ЭТОГО МОЖЕТ СЛУЖИТЬ КОЛЛЕКТИВНОЕ ИССЛЕДОВАНИЕ ИНФЕКЦИОННОЙ ЗАБОЛЕВАЕМОСТИ В СССР В 1919-1990 ГГ., ВЫПОЛНЕННОЕ СОТРУ ДНИКАМИ НАЦИОНАЛЬНОГО НИИ ОБЩЕСТВЕННОГО ЗДОРОВЬЯ ИМ. Н.А. СЕМАШКО. АВТОРЫ НА ОСНОВЕ РАССЕКРЕЧЕННЫХ И НЕИССЛЕДОВАННЫХ АРХИВНЫХ ДОКУМЕНТОВ, А ТАКЖЕ ПУБЛИКАЦИЙ ПОД ГРИФОМ «ДЛЯ СЛУЖЕБНОГО ПОЛЬЗОВАНИЯ» КРИТИКУЮТ ИСТОРИОГРАФИЧЕСКОЕ ПРЕДСТАВЛЕНИЕ О СТОЙКОМ ЭПИДЕМИЧЕСКОМ

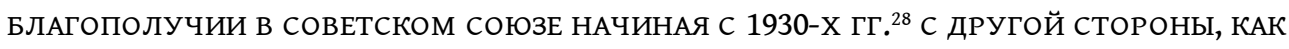
УБЕДИТЕЛЬНО ДЕМОНСТРИРУЕТ ДЖОН ДЭВИС, УСПЕХИ В БОРЬБЕ С ЭПИДЕМИЯМИ ВСЕТАКИ БЫЛИ. ИСТОРИК ПОКАЗЫВАЕТ, ЧТО В ГОДЫ ГРАЖДАНСКОЙ ВОЙНЫ СОВЕТСКИЕ ВЛАСТИ УДАЧНО ВОСПОЛЬЗОВАЛИСЬ ИМПЕРСКОЙ ИНФРАСТРУКТУРОЙ, А ТАКЖЕ 
КОМПЕТЕНЦИЯМИ ПОДГОТОВЛЕННЫХ В ЦАРСКОЕ ВРЕМЯ ВРАЧЕЙ И БАКТЕРИОЛОГОВ, ДЛЯ БОРЬБЫ С ХОЛЕРОЙ. САНИТАРНОЕ ПРОСВЕЩЕНИЕ, НАДЗОР И ВАКЦИНАЦИИ, УТВЕРЖДАЕТ ДЭВИС, ПОМОГЛИ РУКОВОДСТВУ СТРАНЫ СИЛЬНО УМЕНЬШИТЬ ЧИСЛО ЗАРАЖЕНИЙ ХОЛЕРОЙ К ПЕРВОЙ ПОЛОВИНЕ 1920-Х ГОДОВ ${ }^{29}$.

СОВМЕСТНАЯ СТАТЬЯ ЕЛЕНЫ ВИШЛЕНКОВОЙ, СЕРГЕЯ ЗАТРАВКИНА И ЕЛЕНЫ ШЕРСТНЕВОЙ О ДОВОЕННОЙ РЕФОРМЕ ЗДРАВООХРАНЕНИЯ ДЕМОНСТРИРУЕТ, ЧТО ЗА ПРЕДВОЕННОЕ ДЕСЯТИЛЕТИЕ СОВЕТСКАЯ МЕДИЦИНА НАРАСТИЛА КОЛИЧЕСТВЕННЫЕ ПОКАЗАТЕЛИ ЧИСЛА БОЛЬНИЦ, КОЕК И МЕДИЦИНСКОГО ПЕРСОНАЛА, В ТО ВРЕМЯ КАК КАЧЕСТВО МЕДИЦИНСКОЙ ПОМОЩИ ОСТАВАЛОСЬ НА НИЗКОМ УРОВНЕ. К ТОМУ ЖЕ, УТВЕРЖДАЮТ ИССЛЕДОВАТЕЛИ, В 1930-Х МЕДИЦИНСКАЯ ПОМОЩЬ БЫЛА ПЕРЕОРИЕНТИРОВАНА С ОБЩЕДОСТУПНОСТИ НА ВЕДУЩИЕ ОТРАСЛИ ПРОМЫШЛЕННОСТИ ${ }^{30}$. СХОЖИЕ ТЕЗИСЫ НА МАТЕРИАЛАХ ПЕТРОГРАДА/ЛЕНИНГРАДА ВЫДВИГАЕТ КРИСТОФЕР УИЛЬЯМС. ИСТОРИК СЧИТАЕТ, ЧТО РЕВОЛЮЦИОННОЕ ОБЕЩАНИЕ УЛУЧШИТЬ МЕДИЦИНСКУЮ ПОМОЩЬ ШИРОКИМ СЛОЯМ НАСЕЛЕНИЯ ТАК И НЕ БЫЛО ВЫПОЛНЕНО К НАЧАЛУ ВТОРОЙ МИРОВОЙ ВОЙНЫ. НЕХВАТКА РЕСУРСОВ И ЧАСТЫЕ СМЕНЫ ПОЛИТИЧЕСКОГО ВЕКТОРА ОСЛАБЛЯЛИ ЗДОРОВЬЕ ЖИТЕЛЕЙ ГОРОДА И ПРИЛЕГАВШЕГО К НЕМУ РЕГИОНА ${ }^{31}$. КАК СООБЩАЕТ НЕДАВНЯЯ КОЛЛЕКТИВНАЯ СТАТЬЯ СИБИРСКИХ ИСТОРИКОВ, ТАКАЯ ЖЕ СИТУАЦИЯ НАБЛЮДАЛАСЬ В ДАЛЕКИХ ОТ ЛЕНИНГРАДА И МОСКВЫ РЕГИОНАХ, ВО ВСЯКОМ СЛУЧАЕ, В 1920-Х ГГ. ОПИРАЯСЬ НА ОПУБЛИКОВАННЫЕ ДОКУМЕНТЫ И ИСТОЧНИКИ ИЗ ГОСУДАРСТВЕННОГО АРХИВА КРАСНОЯРСКОГО КРАЯ, АВТОРЫ ДОКАЗЫВАЮТ, ЧТО КАЧЕСТВО МЕДИЦИНСКИХ УСЛУГ ОСТАВАЛОСЬ КРАЙНЕ НИЗКИМ НА ПРОТЯЖЕНИИ 1920-Х ГГ., А РАЗНИЦА В ИХ ДОСТУПНОСТИ МЕЖДУ ГОРОДОМ И ДЕРЕВНЕЙ ЛИКВИДИРОВАНА НЕ БЫЛА ${ }^{32}$.

СОМНЕНИЯ В УСПЕХАХ СОВЕТСКОЙ МЕДИЦИНЫ ВЫСКАЗЫВАЮТ И ИССЛЕДОВАТЕЛИ ОТДЕЛЬНЫХ ЛЕКАРСТВ. РАССМОТРЕВШИЙ БОРЬБУ СОВЕТСКИХ ВЛАСТЕЙ С ТУБЕРКУЛЕЗОМ В 1925-1941 ГГ. МАЙКЛ ЗДЕНЕК ДЭВИД УКАЗЫВАЕТ, ЧТО ПРЕДЛОЖЕННЫЙ В ТЕ ГОДЫ ПЛАН МАССОВОГО ПРИВИВАНИЯ НОВОРОЖДЕННЫХ СОВЕТСКИМ ПРЕПАРАТОМ БЦЖ БЫЛ ТЕОРЕТИЧЕСКИ ВЫПОЛНИМ, НО НА ПРАКТИКЕ ЕГО РЕАЛИЗАЦИЯ СТОЛКНУЛАСЬ С ОГРАНИЧЕННЫМ ЧИСЛОМ КВАЛИФИЦИРОВАННЫХ СПЕЦИАЛИСТОВ В РЕГИОНАХ. В ИТОГЕ ВАКЦИНАЦИЯ КОНЦЕНТРИРОВАЛАСЬ В ПЕРВУЮ ОЧЕРЕДЬ ВОКРУГ КРУПНЫХ ПРОМЫШЛЕННЫХ ЦЕНТРОВ ${ }^{33}$. ПОКАЗЫВАЕТ НЕ САМЫЙ ВЫСОКИЙ УРОВЕНЬ СОВЕТСКИХ ДОСТИЖЕНИЙ В МЕДИЦИНЕ ЧЕРЕЗ ИСТОРИЮ ОТДЕЛЬНОГО ПРЕПАРАТА И ЕЛЕНА ШЕРСТНЕВА, ИЗУЧИВШАЯ ПРОИЗВОДСТВО СОВЕТСКОГО ПЕНИЦИЛЛИНА. НА ОСНОВАНИИ АРХИВНЫХ ДОКУМЕНТОВ ОНА ДОКАЗЫВАЕТ, ЧТО ОБЪЕМЫ ПРОИЗВОДСТВА ПЕНИЦИЛЛИНА В СССР БЫЛИ НИЗКИМИ И НЕ СООТВЕТСТВОВАЛИ ПОТРЕБНОСТЯМ СИСТЕМЫ ЗДРАВООХРАНЕНИЯ, А ТЕХНОЛОГИЯ ПОЛУЧЕНИЯ ПРЕПАРАТА ДОЛГОЕ ВРЕМЯ ОСТАВАЛАСЬ УСТАРЕВШЕЙ НА ПОДАВЛЯЮЩЕМ БОЛЬШИНСТВЕ ПРЕДПРИЯТИЙ ${ }^{34}$.

\section{Региональная специфика советской медицины}

ВНИМАНИЕ УЧЕНЫХ ПРИВЛЕКАЕТ РОЛЬ РАЗНОРОДНОСТИ ПРОСТРАНСТВА СОВЕТСКОГО СОЮЗА В МЕДИЦИНЕ. ЯРКИМ ПРЕДСТАВИТЕЛЕМ ЭТОГО ИССЛЕДОВАТЕЛЬСКОГО НАПРАВЛЕНИЯ МОЖНО СЧИТАТЬ ВСЕВОЛОДА БАШКУЕВА, КОТОРЫЙ ПОДРОБНО АНАЛИЗИРУЕТ РАБОТУ СОВЕТСКОЙ МЕДИЦИНЫ В ДОВОЕННОЙ БУРЯТ-МОНГОЛИИ. АВТОР ПОМЕЩАЕТ МЕДИЦИНУ В КОНТЕКСТ ВЛАСТНЫХ ОТНОШЕНИЙ И 
НАЦИЕСТРОИТЕЛЬСТВА. ОН ПОКАЗЫВАЕТ, КАК ПРИ ПОМОЩИ АКТУАЛЬНЫХ ДЛЯ ТЕХ ЛЕТ ЕВГЕНИЧЕСКИХ ПРЕДСТАВЛЕНИЙ БОЛЬШЕВИКИ СТАРАЛИСЬ ТРАНСФОРМИРОВАТЬ РЕГИОН И ИЗУЧАЛИ ЕГО НАСЕЛЕНИЕ ${ }^{35}$. УСТАНОВКУ АЛЬЯНСА ВРАЧЕЙ И БИОЛОГОВ НА ИЗМЕНЕНИЕ УКЛАДА ЖИЗНИ НАЦИОНАЛЬНЫХ ОКРАИН ЧЕРЕЗ МЕДИЦИНУ ТАКЖЕ ОТМЕЧАЕТ ДМИТРИЙ МИХЕЛЬ. В СТАТЬЕ О БОРЬБЕ С ЧУМОЙ ИСТОРИК УТВЕРЖДАЕТ, ЧТО В 1917-1925 ГГ. ПРОИСХОДИЛ ЭКСПОРТ МЕДИЦИНСКОГО ЗНАНИЯ В ЮГОВОСТОЧНУЮ ЧАСТЬ ЕВРОПЕЙСКОЙ ТЕРРИТОРИИ РОССИИ И СОСЕДНИЕ РЕГИОНЫ С КОЧЕВЫМ НАСЕЛЕНИЕМ ${ }^{36}$. С ПОХОЖЕЙ ОПТИКОЙ К ИЗУЧЕНИЮ ИССЛЕДОВАНИЙ МАЛЯРИИ В СОВЕТСКОМ АЗЕРБАЙДЖАНЕ ПОДХОДИТ МАТТИАС БРАУН, КОТОРЫЙ РАССМАТРИВАЕТ ГЕОГРАФИЧЕСКИЕ РЕПРЕЗЕНТАЦИИ ЭТОЙ БОЛЕЗНИ В КАЧЕСТВЕ ОСНОВЫ ДЛЯ УПРАВЛЕНИЯ ТЕРРИТОРИЯМИ ${ }^{37}$. ВМЕСТЕ С ТЕМ, КАК ПОКАЗЫВАЕТ ФЕДОР СИНИЦЫН, РЕГИОНАЛЬНЫЕ КОНТЕКСТЫ МЕДИЦИНЫ В СОВЕТСКОМ СОЮЗЕ НЕ СВОДИЛИСЬ ИСКЛЮЧИТЕЛЬНО К ИМПЛАНТАЦИИ МОДЕРНЫХ МЕДИЦИНСКИХ ПРАКТИК В РАЗНЫХ ЧАСТЯХ СТРАНЫ. ИСТОРИК ОБРАЩАЕТ ВНИМАНИЕ НА ВЗАИМНОЕ УСВОЕНИЕ ЗНАНИЯ МЕЖДУ ТИБЕТСКОЙ И СОВЕТСКОЙ МЕДИЦИНАМИ В БУДДИЙСКИХ РЕГИОНАХ СССР В 1920-Е ГГ. ОН УКАЗЫВАЕТ, ЧТО В ЭТОТ ПЕРИОД СОВЕТСКАЯ ВЛАСТЬ ДЕЙСТВОВАЛА ОСТОРОЖНО, НЕ ИМЕЯ РЕСУРСОВ ДЛЯ СОЗДАНИЯ ПОЛНОЦЕННОЙ АЛЬТЕРНАТИВЫ ТРАДИЦИОННЫМ МЕТОДАМ ЛЕЧЕНИЯ. ОДНАКО, КАК УТВЕРЖДАЕТ ИССЛЕДОВАТЕЛЬ, В 1930-Е ГГ. ДАВЛЕНИЕ НА ТИБЕТСКУЮ МЕДИЦИНУ СНАЧАЛА УСИЛИЛОСЬ, А ЗАТЕМ ВЫЛИЛОСЬ В РЕПРЕССИИ И ЕЕ ЛИКВИДАЦИЮ 38.

17 ПОПЫТКИ АНАЛИЗА ЗДРАВООХРАНИТЕЛЬНОЙ АНТИБОЛЬШЕВИСТСКИХ ТЕРРИТОРИЙ В ГОДЫ ГРАЖДАНСКОЙ ВОЙНЫ В РОССИИ ТАКЖЕ ПРЕДПРИНИМАЮТСЯ ИССЛЕДОВАТЕЛЯМИ ${ }^{39}$. К СОЖАЛЕНИЮ, ОСУЩЕСТВЛЯТЬ ИХ СЛОЖНЕЕ, ПОСКОЛЬКУ ИСТОРИЯ МЕДИЦИНЫ ПРИ БЕЛЫХ ЗНАЧИТЕЛЬНО ХУЖЕ ОБЕСПЕЧЕНА ИСТОЧНИКАМИ. В ЭТОМ ОТНОШЕНИИ ОСОБЕННО ВАЖНА КНИГА НАТАЛЬИ МИРОНОВОЙ ОБ ЭПИДЕМИИ СЫПНОГО ТИФА ПОСЛЕРЕВОЛЮЦИОННЫХ ЛЕТ. ОНА ОБРАЩАЕТ ВНИМАНИЕ НА ОСОБЕННОСТИ ПРОТЕКАНИЯ ЭПИДЕМИИ В РАЗЛИЧНЫХ ГОРОДАХ И СРАВНИВАЕТ ПОЛОЖЕНИЕ ЧАСТЕЙ СТРАНЫ, КОТОРЫЕ КОНТРОЛИРОВАЛИ КРАСНЫЕ И ИХ ПРОТИВНИКИ. ОДНАКО ОНА КОНЦЕНТРИРУЕТСЯ В ОСНОВНОМ НА РАЗЛИЧИЯХ, УПУСКАЯ ИЗ ВИДУ СХОДСТВА ${ }^{40}$.

\section{Советская медицина в транснациональном контексте}

ИССЛЕДОВАТЕЛИ СОВЕТСКОЙ МЕДИЦИНЫ УДЕЛЯЮТ ВНИМАНИЕ НЕ ТОЛЬКО ВНУТРЕННИМ СЮЖЕТАМ, НО И ЕЕ ИНОСТРАННЫМ КОНТАКТАМ. ТАК, В КОЛЛЕКТИВНОЙ СТАТЬЕ МОСКОВСКИХ ИСТОРИКОВ БЫЛИ ОСВЕЩЕНЫ РАЗЛИЧНЫЕ ФОРМЫ СОТРУДНИЧЕСТВА СССР С ДРУГИМИ СТРАНАМИ В СФЕРЕ МЕДИЦИНЫ ${ }^{41}$. НА КОНКРЕТНЫХ СЛУЧАЯХ ЭТОЙ КООПЕРАЦИИ С ВОСТОЧНЫМИ СОСЕДЯМИ ФОКУСИРУЕТСЯ ВСЕВОЛОД БАШКУЕВ. ОН АНАЛИЗИРУЕТ ТО, КАК СОВЕТСКАЯ ВЛАСТЬ ПРИ ПОМОЩИ МЕДИЦИНЫ ВЛИЯЛА НА СОСЕДНИЕ ТУВУ И МОНГОЛИЮ В ПЕРВОЙ ПОЛОВИНЕ ХХ В.42 ЗДЕСЬ ВНОВЬ ОБРАЩАЕТ НА СЕБЯ ВНИМАНИЕ ЦИРКУЛЯЦИЯ ЗНАНИЯ. ИЗУЧИВ КОМАНДИРОВКИ СОВЕТСКИХ ВРАЧЕЙ В КНР В 50-Х - 60-Х ГГ. ХХ В., ОЛЬГА НАГОРНЫХ И НАТАЛЬЯ ШОК ПРОДЕМОНСТРИРОВАЛИ, ЧТО РЕЦЕПЦИЯ МЕДИЦИНСКОГО ЗНАНИЯ ПРОИСХОДИЛА НЕ ТОЛЬКО КИТАЙСКОЙ, НО И СОВЕТСКОЙ СТОРОНОЙ ${ }^{43}$. 

ПАВЕЛ РАТМАНОВ ПРОАНАЛИЗИРОВАЛИ СВЯЗИ НАРКОМЗДРАВА С НЕМЕЦКИМИ КОЛЛЕГАМИ. ОНИ ПОКАЗАЛИ, ЧТО СОВЕТСКАЯ СТОРОНА ИСПОЛЬЗОВАЛА МЕДИЦИНСКОЕ ПРЕДСТАВИТЕЛЬСТВО В ГЕРМАНИИ 1920-Х ГГ. ОДНОВРЕМЕННО ДЛЯ РАСПРОСТРАНЕНИЯ СВОИХ ИДЕЙ И ПОЛУЧЕНИЯ АКТУАЛЬНОЙ ИНФОРМАЦИИ О ЗАПАДНОЙ МЕДИЦИНЕ ${ }^{44}$. НО КОНТАКТЫ С НЕМЦАМИ, ТРАДИЦИОННО СИЛЬНЫЕ В РОССИЙСКОЙ НАУКЕ, НЕ БЫЛИ ЕДИНСТВЕННЫМИ. СЮЗАН ГРОСС СОЛОМОН ПРОАНАЛИЗИРОВАЛА РАБОТУ БЮРО ЗАГРАНИЧНОЙ ИНФОРМАЦИИ НАРКОМЗДРАВА И ОСУЩЕСТВЛЕННЫЕ ПРОЕКТЫ ПО СОЗДАНИЮ ДВУЯЗЫЧНЫХ МЕДИЦИНСКИХ ЖУРНАЛОВ В 1920-Х ГГ. ОНА ПРИШЛА К ВЫВОДУ, ЧТО ПОНАЧАЛУ СОВЕТСКАЯ СТОРОНА ДАВАЛА СВОИМ ПРЕДСТАВИТЕЛЯМ ОБЩИЕ ИНСТРУКЦИИ, НО ПОСТЕПЕННО НАЧАЛА УЧИТЫВАТЬ РАЗНИЦУ В РЕАЛИЯХ ДВУХ СТРАН И ИЗМЕНИЛА СВОЙ ПОДХОД. ТЕМ НЕ МЕНЕЕ, СОГЛАСНО ГРОСС СОЛОМОН, РУКОВОДСТВО НАРКОМЗДРАВА НЕ ОСОЗНАВАЛО ДВУХ ВАЖНЫХ ФАКТОРОВ: ВЛИЯНИЯ, КОТОРОЕ ОКАЗЫВАЛА НА КООПЕРАЦИЮ НОРМАЛИЗАЦИЯ ПОЛИТИЧЕСКОЙ ОБСТАНОВКИ ВО ФРАНЦИИ И ГЕРМАНИИ, А ТАКЖЕ РОЛИ РАЗДЕЛЕНИЯ МЕДИЦИНЫ И ЗДРАВООХРАНЕНИЯ В ЭТИХ СТРАНАХ ${ }^{45}$. ПРОИСХОДИЛО И ЭПИЗОДИЧЕСКОЕ СОТРУДНИЧЕСТВО СОВЕТСКИХ МЕДИКОВ С АМЕРИКАНСКИМИ КОЛЛЕГАМИ. ОДИН ИЗ ПЕРИОДОВ ТАКОГО ВЗАИМОДЕЙСТВИЯ ПОСРЕДСТВОМ ИЗУЧЕНИЯ ПРОЕКТА ПО СОЗДАНИЮ АМЕРИКАНСКОГО ГОСПИТАЛЯ В МОСКВЕ В 1917-1922 ГГ. РАССМОТРЕЛА СЮЗАН ГРАНТ, ПОКАЗАВШАЯ ЛОКАЛЬНЫЕ И ГЛОБАЛЬНЫЕ КОНТЕКСТЫ ЭТОГО ПРЕДПРИЯТИЯ ${ }^{46}$. ДРУГОЙ ПРИМЕР ТАКОЙ КООПЕРАЦИИ ПРОАНАЛИЗИРОВАН ПАВЛОМ РАТМАНОВЫМ И ВСЕВОЛОДОМ БАШКУЕВЫМ СКВОЗЬ ПРИЗМУ ЖУРНАЛА «АМЕРИКАНСКИЙ ОБЗОР СОВЕТСКОЙ МЕДИЦИНЫ», КОТОРЫЙ СУЩЕСТВОВАЛ С 1943 ПО 1948 ГГ. ОН, В ОТЛИЧИЕ ОТ ДРУГИХ ПОДОБНЫХ ИЗДАНИЙ, ЧАСТО СФОРМИРОВАННЫХ ПО ВЗАИМНОЙ ИНИЦИАТИВЕ ДВУХ СТОРОН, БЫЛ СОЗДАН ВЛИЯТЕЛЬНЫМ ИСТОРИКОМ МЕДИЦИНЫ ГЕНРИ (АНРИ) СИГЕРИСТОМ, КОТОРЫЙ ТОГДА РАБОТАЛ В США И СИМПАТИЗИРОВАЛ СОВЕТСКОЙ МОДЕЛИ ЗДРАВООХРАНЕНИЯ ${ }^{47}$. ВАЖНЫЕ РАБОТЫ О МЕДИЦИНСКИХ СВЯЗЯХ ВНУТРИ СОЦИАЛИСТИЧЕСКОГО БЛОКА В ГОДЫ ПОСЛЕ ВТОРОЙ МИРОВОЙ ВОЙНЫ. ПРИМЕРОМ МОЖЕТ СЛУЖИТЬ СТАТЬЯ ЭМЫ ХРЕШАНОВОЙ И ПОЛЫ МАЙКЛС ОБ ИЗУЧЕНИИ РОДОВЫХ БОЛЕЙ В СССР И ЧЕХОСЛОВАКИИ. ИССЛЕДОВАТЕЛЬНИЦЫ УБЕДИТЕЛЬНО ПОКАЗЫВАЮТ, ЧТО В ОБЕИХ СТРАНАХ ДЛЯ ОБЛЕГЧЕНИЯ ЭТИХ СТРАДАНИЙ ИСПОЛЬЗОВАЛАСЬ ПСИХОПРОФИЛАКТИКА. ОДНАКО, НЕСМОТРЯ НА ТО, ЧТО ПРИМЕНЕНИЕ ЭТОГО МЕТОДА В ЧЕХОСЛОВАКИИ МОЖНО СВЯЗАТЬ С СОВЕТСКИМ ВЛИЯНИЕМ, ЕГО СУДЬБА В ДВУХ ГОСУДАРСТВАХ БЫЛА РАЗНОЙ. ВМЕСТЕ С ТЕМ КАК ЧЕХОСЛОВАЦКИЕ, ТАК И СОВЕТСКИЕ ВРАЧИ БЫЛИ ВОВЛЕЧЕНЫ В ТРАНСНАЦИОНАЛЬНЫЕ ДЕБАТЫ О ПСИХОПРОФИЛАКТИКЕ ${ }^{48}$. СОЗВУЧНЫЕ ИДЕИ ПРЕДЛАГАЕТ ДОРА

ВАРГА В СТАТЬЕ О БОРЬБЕ С ПОЛИОМИЕЛИТОМ ВО ВТОРОЙ ПОЛОВИНЕ ХХ В. ОНА ДОКАЗЫВАЕТ, ЧТО УЧЕНЫЕ ИЗ СТРАН СОЦИАЛИСТИЧЕСКОГО БЛОКА (СССР, ЧЕХОСЛОВАКИЯ, ВЕНГРИЯ И КУБА) ИГРАЛИ НЕ МЕНЕЕ ВАЖНУЮ РОЛЬ В ЭТОМ ПРОЦЕССЕ, ЧЕМ РАЗРАБОТАВШИЙ ВАКЦИНУ АМЕРИКАНЕЦ АЛЬБЕРТ СЕЙБИН ${ }^{49}$.

ВАЖНОЕ НАПРАВЛЕНИЕ ДЛЯ ИССЛЕДОВАТЕЛЕЙ СССР - ЭТО ПОПЫТКИ ПОМЕСТИТЬ СОВЕТСКИЙ СОЦИАЛИЗМ В КОНТЕКСТ ИЗУЧЕНИЯ МОДЕРНЫХ ГОСУДАРСТВ. СОЦИАЛЬНАЯ ПОЛИТИКА В ЦЕЛОМ И МЕДИЦИНА В ЧАСТНОСТИ ЯВЛЯЮТСЯ АРГУМЕНТАМИ В ЭТОЙ ДИСКУССИИ. УДАЧНЫМ ОБОБЩЕНИЕМ ВЗГЛЯДОВ ИСТОРИКОВ, 
КОТОРЫЕ ВПИСЫВАЮТ СОВЕТСКУЮ МЕДИЦИНУ В ПРАКТИКУ ФУНКЦИОНИРОВАНИЯ ГОСУДАРСТВ ХХ В. ПОСРЕДСТВОМ КОНЦЕПЦИИ МНОЖЕСТВЕННЫХ МОДЕРНОСТЕЙ, ЯВЛЯЕТСЯ НЕДАВНО ПЕРЕВЕДЕННАЯ НА РУССКИЙ ЯЗЫК КНИГА ДЭВИДА ХОФФМАННА. В НЕЙ СРЕДИ ПРОЧЕГО УБЕДИТЕЛЬНО ДОКАЗЫВАЕТСЯ, ЧТО, НЕСМОТРЯ НА СУЩЕСТВЕННЫЕ ОСОБЕННОСТИ, СОВЕТСКОЕ ЗДРАВООХРАНЕНИЕ БЫЛО ОЧЕНЬ ПОХОЖЕ НА ИНОСТРАННЫЕ АНАЛОГИ

\section{Медицина в ГУЛАГе}

ОДНОЙ ИЗ ОЧЕВИДНЫХ ОТЛИЧИТЕЛЬНЫХ ЧЕРТ СОВЕТСКОГО РЕЖИМА ПО СРАВНЕНИЮ С ДРУГИМИ ГОСУДАРСТВАМИ ТЕХ ЛЕТ БЫЛА МАСШТАБНАЯ СИСТЕМА ИСПРАВИТЕЛЬНОТРУДОВЫХ ЛАГЕРЕЙ. МЕДИЦИНСКИЕ АСПЕКТЫ РАБОТЫ ГУЛАГА ЯВЛЯЮТ СОБОЙ ОДНУ ИЗ АКТУАЛЬНЫХ ТЕМ ПОСЛЕДНИХ ИССЛЕДОВАНИЙ. ИСТОРИК ОКСАНА ЕРМОЛАЕВА НА ОСНОВЕ АРХИВНОГО ДЕЛОПРОИЗВОДСТВА, ЛАГЕРНОЙ ПРЕССЫ И МЕМУАРОВ АНАЛИЗИРУЕТ МЕДИЦИНУ ЛАГЕРНОЙ СИСТЕМЫ 1920-Х - 1930-Х ГГ. ОНА ПРИХОДИТ К ВЫВОДУ, ЧТО НЕВОЗМОЖНОСТЬ РЕШИТЬ САНИТАРНЫЕ ПРОБЛЕМЫ ПРИ ПОМОЩИ СОВРЕМЕННОГО МЕДИЦИНСКОГО ЗНАНИЯ ТОЛКАЛА РУКОВОДИТЕЛЕЙ ЛАГЕРЕЙ НА ДОПОЛНИТЕЛЬНОЕ УВЕЛИЧЕНИЕ КОНТРОЛЯ И НАСИЛИЯ. ЕРМОЛАЕВА ТАКЖЕ УТВЕРЖДАЕТ, ЧТО МЕДИЦИНСКАЯ ИНФОРМАЦИЯ КАК В МЕСТА ЗАКЛЮЧЕНИЯ, ТАК И ИЗ НИХ ДОХОДИЛА В ХАОТИЧНОМ И НЕРЕГУЛЯРНОМ ВИДЕ. БОЛЬШИНСТВО МЕДИЦИНСКИХ ЧАСТЕЙ ОТДАЛЕННЫХ ЛАГЕРНЫХ ПУНКТОВ ОСТАВАЛОСЬ В СОСТОЯНИИ НЕДОФИНАНСИРОВАНИЯ И МАЛО ЧЕМ ПОМОГАЛО ЗАБОЛЕВШИМ. ОДНАКО К КОНЦУ З0-Х ГГ. МЕДИЦИНСКИЕ ПОДРАЗДЕЛЕНИЯ НЕКОТОРЫХ КРУПНЫХ ЛАГЕРЕЙ БЫЛИ НЕПЛОХО ОБЕСПЕЧЕНЫ РЕСУРСАМИ, ЛЕЧИЛИ НЕ ТОЛЬКО ОХРАНУ И ЗАКЛЮЧЕННЫХ, НО И МЕСТНОЕ НАСЕЛЕНИЕ, А ТАКЖЕ ПРОВОДИЛИ ИССЛЕДОВАНИЯ ПЕЛЛАГРЫ, ЦИНГИ И ПСИХИЧЕСКИХ ЗАБОЛЕВАНИЙ ${ }^{51}$. НО ВЫСКАЗЫВАЮТСЯ И СОМНЕНИЯ В КАЧЕСТВЕ ЛАГЕРНЫХ МЕДИЦИНСКИХ ИССЛЕДОВАНИЙ. ИХ ВЫДВИНУЛА В ЧАСТНОСТИ ГОЛЬФО АЛЕКСОПУЛОС, КОТОРАЯ ОТМЕТИЛА, ЧТО ВРАЧИ ВО МНОГОМ ЯВЛЯЛИСЬ ЗАЛОЖНИКАМИ СИТУАЦИИ И БЫЛИ ВЫНУЖДЕНЫ ПРЕЗЕНТОВАТЬ РЕЗУЛЬТАТЫ, ВПИСЫВАВШИЕСЯ В ОФИЦИАЛЬНЫЕ ПОЗИТИВНЫЕ ПРЕДСТАВЛЕНИЯ О СИТУАЦИИ В ГУЛАГЕ ${ }^{52}$. ВАЖНО ОТМЕТИТЬ, ЧТО ИССЛЕДОВАНИЯ ЭТОЙ ТЕМЫ НЕ СВОДЯТСЯ ИСКЛЮЧИТЕЛЬНО К ТОМУ, ЧТО ПРОИСХОДИЛО ВНУТРИ ЛАГЕРНЫХ СТЕН. ДЭН ХИЛИ, ИССЛЕДУЯ ИНВАЛИДНОСТЬ В ГУЛАГЕ, УТВЕРЖДАЕТ, ЧТО В ПЕРИОД СТАЛИНИЗМА ПОЛИТИКА ПО ОТНОШЕНИЮ К ЛЮДЯМ С ОГРАНИЧЕННЫМИ ВОЗМОЖНОСТЯМИ ЛАГЕРЯХ ПРЕДСТАВЛЯЛА СОБОЙ МИНИАТЮРНУЮ И ПРЕСТУПНУЮ ВЕРСИЮ ТАКОВОЙ ЗА ПРЕДЕЛАМИ МЕСТ ЛИШЕНИЯ СВОБОДЫ ${ }^{53}$.

\section{Заключение}

МОЖНО УТВЕРЖДАТЬ, ЧТО К 2020 Г. ИСТОРИЯ СОВЕТСКОЙ МЕДИЦИНЫ ПРЕДСТАВЛЯЕТ СОБОЙ ДИНАМИЧНО РАЗВИВАЮЩЕЕСЯ ТРАНСНАЦИОНАЛЬНОЕ ИССЛЕДОВАТЕЛЬСКОЕ ПОЛЕ, В КОТОРОМ ТРУДЯТСЯ И ВЗАИМОДЕЙСТВУЮТ ПРЕДСТАВИТЕЛИ РАЗНЫХ ПОДХОДОВ И ИНСТИТУЦИЙ. ТРАДИЦИОННЫЕ ДЛЯ ЭТОГО ПОЛЯ ИССЛЕДОВАНИЯ БИОГРАФИЙ, НАУЧНЫХ ШКОЛ И УЧРЕЖДЕНИЙ ПРОДОЛЖАЮТ ЗАНИМАТЬ СВОЮ ЗАМЕТНУЮ НИШУ. ВМЕСТЕ С ТЕМ РАЗВИВАЮТСЯ ПРОБЛЕМАТИКИ, ПОДНЯТЫЕ ИССЛЕДОВАТЕЛЯМИ В ПОСЛЕДНЕЙ ЧЕТВЕРТИ ХХ В. ВО-ПЕРВЫХ, РЕЧЬ ИДЕТ ОБ 
ИЗУЧЕНИИ ОТНОШЕНИЙ СОВЕТСКИХ ВРАЧЕЙ С ПОЛИТИКАМИ И АНАЛИЗЕ ВЛАСТНОГО ХАРАКТЕРА СОВЕТСКОГО МЕДИЦИНСКОГО ДИСКУРСА; ВО-ВТОРЫХ, О ПРОБЛЕМЕ ЭКСПЕРТНОГО СТАТУСА ВРАЧЕЙ В СССР, ТЕСНО СВЯЗАННОЙ С ИХ ОТНОШЕНИЯМИ С ФЕЛЬДШЕРАМИ И МЕДИЦИНСКИМИ СЕСТРАМИ. РЯД ПУБЛИКАЦИЙ ПОСЛЕДНЕГО ДЕСЯТИЛЕТИЯ ПОДНЯЛ ВОПРОС ОБ УСПЕШНОСТИ СОВЕТСКОГО ЗДРАВООХРАНЕНИЯ И СТАЛ РЕАКЦИЕЙ НА ДОМИНИРОВАВШИЙ В СССР И НЕКОТОРОЕ ВРЕМЯ ПОСЛЕ ЕГО КРАХА ИСТОРИКО-МЕДИЦИНСКИЙ КАНОН ${ }^{54}$. ПОЖАЛУЙ, НАИБОЛЕЕ АКТИВНО РАЗВИВАЮТСЯ ИССЛЕДОВАНИЯ РЕГИОНАЛЬНЫХ ОСОБЕННОСТЕЙ СОВЕТСКОЙ МЕДИЦИНЫ, А ТАКЖЕ ПОПЫТКИ ВПИСАТЬ ЕЕ В ШИРОКИЙ ТРАНСНАЦИОНАЛЬНЫЙ КОНТЕКСТ. ЭТО МОЖНО СВЯЗАТЬ С ИНТЕРЕСОМ К УКАЗАННЫМ ТЕМАМ В СОВРЕМЕННОЙ РУСИСТИКЕ В ЦЕЛОМ. ПЕРСПЕКТИВНЫМ ПРЕДСТАВЛЯЕТСЯ УГЛУБЛЕННОЕ ИССЛЕДОВАНИЕ ЗДРАВООХРАНИТЕЛЬНЫХ ПРАКТИК КРАСНЫХ И БЕЛЫХ В ГОДЫ ГРАЖДАНСКОЙ ВОЙНЫ. ИХ СХОДСТВА МОГУТ БЫТЬ ОСНОВОЙ ДЛЯ ДАЛЬНЕЙШИХ РАССУЖДЕНИЙ О СПЕЦИФИЧЕСКИХ ПРАКТИКАХ УПРАВЛЕНИЯ В УСЛОВИЯХ МАСШТАБНОГО СОЦИАЛЬНОПОЛИТИЧЕСКОГО КРИЗИСА И ЭПИДЕМИИ. НАКОНЕЦ, ПУБЛИКАЦИИ О МЕДИЦИНЕ В ИСПРАВИТЕЛЬНО-ТРУДОВЫХ ЛАГЕРЯХ ПРЕДСТАВЛЯЮТ ЕЩЕ ОФОРМЛЯЮЩЕЕСЯ НАПРАВЛЕНИЕ ИССЛЕДОВАНИЙ В ИСТОРИОГРАФИИ СОВЕТСКОЙ МЕДИЦИНЫ. ЧТО КАСАЕТСЯ ХРОНОЛОГИЧЕСКИХ РАМОК ИССЛЕДОВАНИЙ ПОСЛЕДНЕГО ДЕСЯТИЛЕТИЯ, ТО ЗДЕСЬ МОЖНО УВИДЕТЬ ОПРЕДЕЛЕННОЕ СМЕЩЕНИЕ. НА РУБЕЖЕ ХХ И ХХІ ВВ. ИСТОРИКИ СОВЕТСКОЙ МЕДИЦИНЫ В ОСНОВНОМ ОБРАЩАЛИСЬ К ПЕРИОДУ ДО ВТОРОЙ МИРОВОЙ ВОЙНЫ. СЕЙЧАС ЖЕ МНОГИЕ СПЕЦИАЛИСТЫ, В ТОМ ЧИСЛЕ И ТЕ, КТО ИЗВЕСТЕН БЛАГОДАРЯ ПУБЛИКАЦИЯМ О ДОВОЕННОЙ СОВЕТСКОЙ МЕДИЦИНЕ, ОБРАЩАЮТСЯ И К 1939-1989 ГГ. ПРЕЖДЕ ВСЕГО ЭТО КАСАЕТСЯ ТРАДИЦИОННЫХ ИСТОРИКО-МЕДИЦИНСКИХ ТЕМАТИК И ИЗУЧЕНИЯ ВЗАИМОДЕЙСТВИЯ СОВЕТСКОЙ МЕДИЦИНЫ С ЗАРУБЕЖЬЕМ.

\section{NOTES}

1. СТАТЬЯ ПОДГОТОВЛЕНА ПРИ ПОДДЕРЖКЕ РОССИЙСКОГО НАУЧНОГО ФОНДА, ГРАНТ №19-48-04110. The article was prepared within the project № 19-48-04110 supported by Russian Science Foundation.

А. РЕННЕР, «ИССЛЕДОВАНИЯ ИСТОРИИ МЕДИЦИНЫ XVIII-XIX ВЕКОВ НА ЗАПАДЕ: НОВЫЕ ПОДХОДЫ И ПЕРСПЕКТИВЫ», В Л.А. БУЛГАКОВА, РЕД., МЕДИЦИНА РОССИИ В ГОДЫ ВОЙНЫ И МИРА: НОВЫЕ ДОКУМЕНТЫ И ИССЛЕДОВАНИЯ СПБ.: НЕСТОР-ИСТОРИЯ, 2011, С. 215. О ДЕЛЕНИИ ИСТОРИИ МЕДИЦИНЫ НА ТРАДИЦИОННУЮ (ИЗУЧАЮЩУЮ ПОСЛЕДОВАТЕЛЬНОЕ ПРИРАЩЕНИЕ МЕДИЦИНСКОГО ЗНАНИЯ И ИСТОРИЮ РАЗВИТИЯ ВРАЧЕБНОЙ ПРОФЕССИИ) И СОЦИАЛЬНУЮ/ НОВУЮ (СФОКУСИРОВАННУЮ НА РАЗНООБРАЗИИ МЕДИЦИНЫ, КОНКУРИРУЮЩИХ ШКОЛАХ, ПАЦИЕНТАХ, ЯЗЫКАХ МЕДИЦИНЫ РАЗЛИЧНЫХ ПЕРИОДОВ И МЕДИЦИНСКОЙ ПРАКТИКЕ) СМ.: А.Э. АФАНАСЬЕВА, «НОВАЯ ИСТОРИЯ МЕДИЦИНЫ В НАЧАЛЕ ХХІ ВЕКА: ОСНОВНЫЕ ТЕНДЕНЦИИ РАЗВИТИЯ», ПРЕПОДАВАТЕЛЬ ХХІ ВЕК, 2016, по. 4-2, С. 486-499; Д.В. МИХЕЛЬ, «СОЦИАЛЬНАЯ ИСТОРИЯ МЕДИЦИНЫ: СТАНОВЛЕНИЕ И ПРОБЛЕМАТИКА», ЖУРНАЛ ИССЛЕДОВАНИЙ СОЦИАЛЬНОЙ ПоЛИТИКИ, 7, 3, 2009, с. 295-307; Ю. ШЛЮМБУМ, М. ХАГНЕР, И. СИРОТКИНА, «ВВЕДЕНИЕ. 
ИСТОРИЯ МЕДИЦИНЫ: АКТУАЛЬНЫЕ ТЕНДЕНЦИИ И ПЕРСПЕКТИВЫ», В Ю. ШЛЮМБУМ, М. ХАГНЕР, И. СИРОТКИНА, РЕД., БОЛЕЗНЬ И ЗДОРОВЬЕ: НОВЫЕ ПОДХОДЫ К ИСТОРИИ МЕДИЦИНЫ СПБ.: ЕВРОПЕЙСКИЙ УНИВЕРСИТЕТ В САНКТ-ПЕТЕРБУРГЕ; АЛЕТЕЙЯ, 2008, С. 8-27.

2. РЕННЕР, «ИССЛЕДОВАНИЯ ИСТОРИИ МЕДИЦИНЫ XVIII-XIX ВЕКОВ НА ЗАПАДЕ...», С. 215. ДЕЙСТВИТЕЛЬНО, ИМЕННО В 2000-Х ГГ. БЫЛ ОПУБЛИКОВАН ЦЕЛЫЙ РЯД ЗНАКОВЫХ РАБОТ ПО TEME. См.: Daniel Beer, Renovating Russia: The Human Sciences and the Fate of Liberal Modernity, 1880-1930, Ithaca: Cornell University Press, 2008; Frances Lee Bernstein, The Dictatorship of Sex: Lifestyle Advice for the Soviet Masses, DeKalb: Northern Illinois University Press, 2007; Susan Gross Solomon, ed., Doing Medicine Together: Germany and Russia Between the Wars, Toronto: University of Toronto Press, 2006; Tricia Starks, The Body Soviet: Propaganda, Hygiene, and the Revolutionary State, Madison: University of Wisconsin Press, 2008.

3. СМ.: П.Э. РАТМАНОВ, «СОВЕТСКОЕ ЗДРАВООХРАНЕНИЕ В ОСВЕЩЕНИИ ЗАПАДНЫХ АВТОРОВ (1920-1960-Е ГГ.)», ВЕСТНИК ОБЩЕСТВЕННОГО ЗДОРОВЬЯ И ЗДРАВООХРАНЕНИЯ ДАЛЬНЕГО ВОСТОКА России, 2013, no. 3, http://www.fesmu.ru/voz/20133/2013307.aspx.

4. См.: Grégory Dufaud, «Médecins, médecine et santé en Union soviétique», Histoire sociale/Social History, 46 (92), 2013, p. 497-507; Susan Gross Solomon, «East European Public Health and the Cold War. In Search of Circulation», Revue D'Études Comparatives Est-Ouest, 1, 2018, p. 7-43.

5. С.П. ГЛЯНЦЕВ, А.А. СТОЧИК, «КАК СОЗДАВАЛСЯ “ВЫСШИЙ ОРГАН МЕДИЦИНСКОЙ МЫСЛИ СТРАНЫ" - АКАДЕМИЯ МЕДИЦИНСКИХ НАУК СССР», ВЕСТНИК РОССИЙСКОЙ АКАДЕМИИ НАУҚ 90 (8), 2020, С. 768-777.

6. М.Н. СВИНЦОВА, «КИРОВСКИЙ ИНСТИТУТ ЭПИДЕМИОЛОГИИ И МИКРОБИОЛОГИИ В ГОДЫ ВЕЛИКОЙ ОТЕЧЕСТВЕННОЙ ВОЙНЫ (1941-1945 ГГ.)», ВОЕННО-МЕДИЦИНСКИЙ ЖУРНАЛ, 339 (4), 2018, С. 85-90.

7. С.Д. БАТОЕВ, «ВКЛАД ПРОФЕССОРА МЕДИЦИНЫ В.М. БРОННЕРА В БОРЬБУ С ВЕНЕРИЧЕСКИМИ ЗАБОЛЕВАНИЯМИ В ЗАБАЙКАЛЬЕ В 1920-Е ГОДЫ», ИСТОРИЯ МЕДИЦИНЫ, 4 (1), 2017, С. 16-26; Е.И. КАЛИКИНСКАЯ, «В.Ф.ВОЙНО-ЯСЕНЕЦКИЙ - ВЕДУЩИЙ ХИРУРГ КРАСНОЯРСКОГО ЭВАКОГОСПИТАЛЯ № 1515 В ГОДЫ ВЕЛИКОЙ ОТЕЧЕСТВЕННОЙ ВОЙНЫ», ВОЕННО-МЕДИЦИНСКИЙ ЖУРНАЛ, 340 (6), 2019, С. 83-89; М.Ш. КНОПОВ, В.К. ТАРНУХА, «ВЫДАЮЩИЙСЯ КАРДИОЛОГ П.Е. ЛУКОМСКИЙ (К 40-ЛЕТИЮ СО ДНЯ КОНЧИНЫ)», КЛИНИЧЕСКАЯ МЕДИЦИНА, 92 (1), 2014, С. 67 69.

8. Е.В. АРСЕНТЬЕВ, В.А. РЕШЕТНИКОВ, «К БИОГРАФИИ Н.А. СЕМАШКО: ДЕЯТЕЛЬНОСТЬ ПЕРВОГО НАРКОМА ЗДРАВООХРАНЕНИЯ В 1920-1925 ГГ.», ИСТОРИЯ МЕДИЦИНЫ, 5 (3), 2018, С. 217-229.

9. Nikolai Krementsov, A Martian Stranded on Earth: Alexander Bogdanov, Blood Transfusions, and Proletarian Science, Chicago: University of Chicago Press, 2011.

10. Daniel Todes, Ivan Pavlov: A Russian Life in Science, Oxford - New York: Oxford University Press, 2014.

11. В.И. БОРОДУЛИН, А.В. ТОПОЛЯНСКИЙ, «К ИСТОРИИ ГАСТРОЭНТЕРОЛОГИИ В СССР: О НАУЧНОЙ ШКОЛЕ М.И. ПЕВЗНЕРА», ПРОБЛЕМЫ СОЦИАЛЬНОЙ ГИГИЕНЫ, ЗДРАВООХРАНЕНИЯ И ИСТОРИИ МЕДИЦИНЫ, 6, 2012, С. 45-51.

12. Р.А. ФАНДО, «МОСКОВСКАЯ ШКОЛА МЕДИЦИНСКОЙ ГЕНЕТИКИ С.Г. ЛЕВИТА», ПРОБЛЕМЫ СОЦИАЛЬНОЙ ГИГИЕНЫ, ЗДРАВООХРАНЕНИЯ И ИСТОРИИ МЕДИЦИНЫ, 22 (1), 2014, С. 44-47.

13. И.В. ЕГОРЫШЕВА, «РЕПРЕССИИ В ОТНОШЕНИИ ВРАЧЕЙ В ГОДЫ ГРАЖДАНСКОЙ ВОЙНЫ», ПРОБЛЕМЫ СОЦИАЛЬНОЙ ГИГИЕНЫ, ЗДРАВООХРАНЕНИЯ И ИСТОРИИ МЕДИЦИНЫ 26 (6), 2018, С. 483486.

14. В. ТОПОЛЯНСКИЙ, «КОНЕЦ ПИРОГОВСКОГО ОБЩЕСТВА», РОССИЯ ХХІ, 2014, no. 4, с. 168-191.

15. Igor J. Polianski, «Bolshevik Disease and Stalinist Terror: On the Historical Casuistry of Artificial Pneumothorax», Medical History, 59 (1), 2015, p. 32-43. 
16. К. УИЛЬЯМС, «КРИЗИС В МЕДИЦИНЕ И СТАЛИНСКИЕ РЕПРЕССИИ ПРОТИВ МЕДИЦИНСКИХ РАБОТНИКОВ В ЛЕНИНГРАДЕ В 1937-1938 ГГ.», ПЕТЕРБ УРГСКИЙ ИСТОРИЧЕСКИЙ ЖУРНАЛ 2019, no. 1, С. $164-186$.

17. Н. ТАМАРУЧИ, «МЕДИЦИНА И ВЛАСТЬ», НОВОЕ ЛИТЕРАТУРНОЕ ОБОЗРЕНИЕ, 2014, no. 3, С. 134155.

18. В.А. ЯКОВЕНКО, «ЗДОРОВЬЕ НАСЕЛЕНИЯ И СОЦИАЛЬНАЯ СТРАТИФИКАЦИЯ В ПУБЛИЦИСТИКЕ Н.А. СЕМАШКО (1918-1928 ГГ.)», РОССИЯ И СОВРЕМЕННЫЙ МИР, 2020, nо. 3 С. 191-203.

19. Tricia A. Starks, «Propagandizing the Healthy, Bolshevik Life in the Early USSR», American Journal of Public Health, 107, 2017, p. 1718-1724.

20. Irina Sirotkina, «Toward a Soviet Psychiatry: War and the Organization of Mental Health Care in Revolutionary Russia», in Frances Lee Bernstein, Christopher Burton, Dan Healey, eds., Soviet Medicine: Culture, Practice, and Science, DeKalb: Northern Illinois University Press, 2010, p. 27-48.

21. Г. ДЮФО, «НОВЫЕ ПОДХОДЫ К СУМАСШЕСТВИЮ: РАЗВИТИЕ ВНЕБОЛЬНИЧНОЙ ПСИХИАТРИИ В СОВЕТСКОЙ РОССИИ В 1920-Е - НАЧАЛЕ 1930-Х ГОДОВ», ИСТОРИЯ МЕДИЦИНЫ, 2 (3), 2015, С. $373-$ 389.

22. М.А. ПОГОРЕЛОВ, «МЕДИКАЛИЗАЦИЯ ПРЕСТУПНОСТИ В СОВЕТСКОЙ СУДЕБНОЙ ПСИХИАТРИИ (1918-1936 ГГ.)», ЖУРНАЛ ИССЛЕДОВАНИЙ СОЦИАЛЬНОЙ ПОЛИТИКИ, 16 (2), 2018, с. 205-220.

23. Pavel A. Vasilyev, «Drug Addiction and the Practice of Public Health in Late Imperial and Early Soviet Russia», ВЕСТНИК САНКТ-ПЕТЕРБУРГСКОГО УНИВЕРСИТЕТА. ИСТОРИЯ 63 (4), 2018, p. 1100-1119.

24. Igor J. Polianski, «Pathologia Religiosa: Medicine and the Anti-religious Movement in the Early Soviet Union», Journal of Contemporary History, 53 (3), 2018, p. 524-549.

25. Benjamin Zajicek, «Soviet Madness: Nervousness, Mild Schizophrenia, and the Professional Jurisdiction of Psychiatry in the USSR, 1918-1936», Ab Imperio, 2014, no. 4, C. 167-194.

26. Samuel C. Ramer, «The Russian Feldsher: A PA Prototype in Transition», Journal of American Academy of Physician Assistants, 31 (11), 2018, p. 1-6.

27. Susan Grant, «Creating Cadres of Soviet Nurses, 1936-1941», in Susan Grant, ed., Russian and Soviet Healthcare from an International Perspective: Comparing Professions, Practice and Gender, 18801960, Cham: Palgrave Macmillan, 2017, p. 57-76.

28. С.Н. ЗАТРАВКИН, Р.У. ХАБРИЕВ, В.О. ЩЕПИН, А.С. САРКИСОВ, «ЗАБОЛЕВАЕМОСТЬ ИНФЕКЦИОННЫМИ БОЛЕЗНЯМИ В СССР: МИФЫ И РЕАЛЬНОСТЬ. СООБЩЕНИЕ 1. 1919-1949 ГОДЫ», ПРОБЛЕМЫ СОЦИАЛЬНОЙ ГИГИЕНЫ, ЗДРАВООХРАНЕНИЯ И ИСТОРИИ МЕДИЦИНЫ 26 (5), 2018, С. 350356; ОНИ ЖЕ, «ЗАБОЛЕВАЕМОСТЬ ИНФЕКЦИОННЫМИ БОЛЕЗНЯМИ В СССР: МИФЫ И РЕАЛЬНОСТЬ. СООБЩЕНИЕ 2. 1950-1990 ГОДЫ», ПРОБЛЕМЫ СОЦИАЛЬНОЙ ГИГИЕНЫ, ЗДРАВООХРАНЕНИЯ И ИСТОРИИ мЕДицины, 26 (6), 2018, с. 465-471.

29. John P. Davis, Russia in the Time of Cholera: Disease under Romanovs and Soviets, London - New York: I.B. Tauris, 2018.

30. С. ЗАТРАВКИН, Е. ВИШЛЕНКОВА, Е. ШЕРСТНЕВА, «“КОРЕННОЙ ПЕРЕЛОМ”: ДОВОЕННАЯ РЕФОРМА СОВЕТСКОГО ЗДРАВООХРАНЕНИЯ», Quaestio Rossica, 8 (2), 2020, с. 652-666.

31. Christopher Williams, Health and Welfare in St. Petersburg, 1900-1941. Protecting the Collective, London - New York: Routledge, 2018.

32. Т.А. КАТЦИНА, И.И. КРЫЛОВ, Н.В. ПАШИНА, Л.Э. МЕЗИТ, «СТАНОВЛЕНИЕ СОВЕТСКОГО ЗДРАВООХРАНЕНИЯ В РОССИЙСКОЙ ПРОВИНЦИИ В 1920-Е ГОДЫ», ПРОБЛЕМЫ СОЦИАЛЬНОЙ ГИГИЕНЫ, ЗДРАВООХРАНЕНИЯ И ИСТОРИИ МЕДИЦИНЫ, 25 (1), 2017, С. 41-45.

33. Michael Zdenek David, «Vaccination Against Tuberculosis with BCG - A Study of Innovation in Soviet Public Health, 1925-1941», in Bernstein, Burton, Healey, Soviet Medicine, p. 132-154.

34. Е.В. ШЕРСТНЕВА, «ОРГАНИЗАЦИЯ ПРОМЫШЛЕННОГО ПРОИЗВОДСТВА ПЕНИЦИЛЛИНА В СССР», ПРОБЛЕМЫ СОЦИАЛЬНОЙ ГИГИЕНЫ, ЗДРАВООХРАНЕНИЯ И ИСТОРИИ МЕДИЦИНЫ 28 (2), 2020, С. 320- 
325; ОНА ЖЕ, «ПРОБЛЕМЫ НАЧАЛЬНОГО ЭТАПА МАССОВОГО ВЫПУСКА ПЕНИЦИЛЛИНА В СССР», ПРОБЛЕМЫ СОЦИАЛЬНОЙ ГИГИЕНЫ, ЗДРАВООХРАНЕНИЯ И ИСТОРИИ МЕДИЦИНЫ 28 (1), 2020, С. $152-$ 157.

35. В.Ю. БАШКУЕВ, «ГЕОПОЛИТИКА И ЕВГЕНИКА В КОНТЕКСТЕ НАУЧНОГО ИЗУЧЕНИЯ БУРЯТМОНГОЛЬСКОЙ АСССР В 1920-Х - НАЧАЛЕ 1930-Х ГГ.», ВЛАСТЬ, 2014, no. 5, С. 140-145; ОН ЖЕ, «СОВЕТСКАЯ СОЦИАЛЬНАЯ ЕВГЕНИКА И НАЦМЕНЬШИНСТВА: ЛИКВИДАЦИЯ СИФИЛИСА В БУРЯТМОНГОЛИИ КАК ЭЛЕМЕНТ ПРОГРАММЫ МОДЕРНИЗАЦИИ НАЦИОНАЛЬНОГО РЕГИОНА (19231930)», ВЛАСТЬ, 2012, no. 10, С. 174-178.

36. Dmitry Mikhel, «Fighting Plague in Southeast European Russia, 1917-1925 - A case Study in Early Soviet Medicine», in Bernstein, Burton, Healey, Soviet Medicine, p. 49-70.

37. Matthias Braun, «From Landscapes to Labscapes: Malaria Research and Anti-Malaria Policy in Soviet Azerbaijan, 1920-41», Jahrbücher für Geschichte Osteuropas, 61 (4), 2013, S. 513-530.

38. Ф.Л. СИНИЦЫН, «СОВЕТСКАЯ ПОЛИТИКА В ОТНОШЕНИИ БУДДИЙСКОЙ СИСТЕМЫ ЗДРАВООХРАНЕНИЯ В 1920-1930-Е ГГ.: РЕФОРМЫ И РЕПРЕССИИ 》, ВЕСТНИК РОССИЙСКОГО УНИВЕРСИТЕТА ДРУЖБЫ НАРОДОВ. СЕРИЯ: ИСТОРИЯ РОССИИ, 2012, no. 2, С. 91-104.

39. В КАЧЕСТВЕ ПРИМЕРОВ СМ.: А.В. ПОСАДСКИЙ, «МЕДИЦИНА БЕЛОГО ЮГА В ГРАЖДАНСКОЙ ВОЙНЕ: СТРУКТУРЫ, РЕШЕНИЯ, ПОВСЕДНЕВНОСТЬ», НОВЕЙШАЯ ИСТОРИЯ РОССИИ, 10 (2), 2020, С. 315-329; В.А. ШАЛАМОВ, «ЗДРАВООХРАНЕНИЕ В ЗАБАЙКАЛЬСКОЙ ОБЛАСТИ ВО ВРЕМЯ РЕЖИМА АТАМАНА Г.М. СЕМЕНОВА (МАЙ 1919-ЯНВАРЬ 1920 Г.)», ВЕСТНИК ТОМСКОГО ГОСУДАРСТВЕННОГО УНИВЕРСИТЕТА. ИСТОРИЯ, 2019, по. 61, 2019, С. 67-77; ОН ЖЕ, «ЗДРАВООХРАНЕНИЕ В ЗАБАЙКАЛЬСКОЙ ОБЛАСТИ ВО ВРЕМЯ РЕЖИМА АТАМАНА Г.М. СЕМЕНОВА (ОСЕНЬ 1918-ВЕСНА 1919 Г.)», ВЕСТНИК ТОМСКОГО ГОСУДАРСТВЕННОГО УНИВЕРСИТЕТА 2017, no. 414, С. 156-166; Vladislav Yakovenko, «Non-Bolshevik Healthcare in Russia: Organization of the Medical Care in the Northern Region (August 1918 - October 1919)», Basic Research Programme. Series HUM «Humanities», 2019, no. 176, https://wp.hse.ru/data/2019/05/27/1494805762/176HUM2019.pdf

40. Н.А. МИРОНОВА, ВЕЛИКАЯ ЭПИДЕМИЯ: СЫПНОЙ ТИФ В РОССИИ В ПЕРВЫЕ ГОДЫ СОВЕТСКОЙ ВЛАСТ,И М.: УНИВЕРСИТЕТ ДМИТРИЯ ПОЖАРСКОГО; РУССКИЙ ФОНД СОДЕЙСТВИЯ ОБРАЗОВАНИЮ И НАУКЕ, 2020.

41. М.Ю. ПОДДУБНЫЙ, И.В. ЕГОРЫШЕВА, А.В. МОРОЗОВ, «МЕЖДУНАРОДНОЕ СОТРУДНИЧЕСТВО НАРКОМАТА ЗДРАВООХРАНЕНИЯ В ПЕРИОД 1920-1930 ГОДОВ», ВЕСТНИК СОВРЕМЕННОЙ КЛИНИЧЕСКОЙ МЕДИЦИНЫ, 10 (5), 2017, С. 74-78.

42. В.Ю. БАШКУЕВ, «МЕДИКО-САНИТАРНЫЕ ЭКСПЕДИЦИИ НАРКОМЗДРАВА РСФСР В ТУВИНСКОЙ НАРОДНОЙ РЕСПУБЛИКЕ И СТАНОВЛЕНИЕ ТУВИНСКОГО ЗДРАВООХРАНЕНИЯ (КОНЕЦ 1920-Х СЕРЕДИНА 1930-Х ГГ.)», ВЕСТНИК ТОМСКОГО ГОСУДАРСТВЕННОГО УНИВЕРСИТЕТА 2018, no. 426, С. 5263; ОН ЖЕ, «ЭКСПОРТ СОВЕТСКОЙ МЕДИЦИНЫ В 1920-Х ГГ.: МЕДИКО-САНИТАРНЫЕ ЭКСПЕДИЦИИ НАРКОМЗДРАВА РСФСР В МОНГОЛЬСКОЙ НАРОДНОЙ РЕСПУБЛИКЕ», ВЛАСТЬ, 24 (6), 2016, С. $196-$ 202.

43. О.С. НАГОРНЫХ, Н.П. ШОК, «КОМАНДИРОВКИ СОВЕТСКИХ ВРАЧЕЙ В КНР В 1950-1960-Е ГГ.: РЕАЛИЗАЦИЯ ПЛАНОВ СОТРУДНИЧЕСТВА В СФЕРЕ МЕДИЦИНЫ И ЗДРАВООХРАНЕНИЯ», ВЕСТНИК ТОМСКОГО ГОСУДАРСТВЕННОГО УНИВЕРСИТЕТА. ИСТОРИЯ, 2020, no. 65, С. 75-85.

44. П.Э. РАТМАНОВ, Ю.В. КИРИК, «ПРЕДСТАВИТЕЛЬСТВО НАРОДНОГО КОМИССАРИАТА ЗДРАВООХРАНЕНИЯ РСФСР В ГЕРМАНИИ (1921-1929 ГГ.) В КОНТЕКСТЕ МЕЖДУНАРОДНЫХ СВЯЗЕЙ СОВЕТСКОЙ РОССИИ», ДАЛЬНЕВОСТОЧНЫЙ МЕДИЦИНСКИЙ ЖУРНАЛ, 2019, no. 4, С. 41-46.

45. Susan Gross Solomon, «Thinking Internationally, Acting Locally: Soviet Public Health as Cultural Diplomacy in the 1920s», in Grant, ed., Russian and Soviet Healthcare from an International Perspective, p. 193-216.

46. Susan Grant, «The American Hospital in Moscow: A Lesson in International Cooperation, 1917-1923», Medical History, 59 (4), 2015, p. 554-574. 
47. П.Э. РАТМАНОВ, В.Ю. БАШКУЕВ, «СОВЕТСКАЯ МЕДИЦИНА НА СТРАНИЦАХ АМЕРИКАНСКОЙ МЕДИЦИНСКОЙ ПЕРИОДИКИ: ЖУРНАЛ “АМЕРИКАНСКИЙ ОБЗОР СОВЕТСКОЙ МЕДИЦИНЫ” (19431948) В КОНТЕКСТЕ ДВУСТОРОННИХ ОТНОШЕНИЙ СССР И США», ПРОБЛЕМЫ СОЦИАЛЬНОЙ ГИГИЕНЫ, ЗДРАВООХРАНЕНИЯ И ИСТОРИИ МЕДИЦИНЫ, 27 (5), 2019, С. 930-935.

48. Ema Hrešanova, Paula A. Michaels, «Investigating Pain in Soviet and Czechoslovakian Maternity Care», Revue d'Études Comparatives Est-Ouest, 1, 2018, p. 45-69.

49. Dora Vargha, «The Socialist World in Global Polio Eradication», Revue d'Études Comparatives Est-Ouest, 1, 2018, p. 71-94.

50. Д.Л. ХОФФМАНН, ВЗРАЩИВАНИЕ МАСС. МОДЕРНОЕ ГОСУДАРСТВО И СОВЕТСКИЙ СОЦИАЛИЗМ. 19141939, М.: НОВОЕ ЛИТЕРАТУРНОЕ ОБОЗРЕНИЕ, 2018.

51. Oxana Ermolaeva, «Health Care, the Circulation of Medical Knowledge, and Research in the Soviet GULAG in the 1930s», East Central Europe, 40, 2013, p. 341-365.

52. Golfo Alexopoulos, «Medical Research in Stalin's Gulag», Bulletin of the History of Medicine, 90 (3), 2016, p. 363-393.

53. Dan Healey, «Lives in the Balance: Weak and Disabled Prisoners and the Biopolitics of the Gulag», Kritika: Explorations in Russian and Eurasian History, 16 (3), 2015, p. 527-556.

54. ЭТОТ КАНОН БЫЛ СФОРМИРОВАН НА РУБЕЖЕ 1940-Х И 1950-Х ГГ. И НОСИЛ СКОРЕЕ ЭПИЧЕСКИЙ, ЧЕМ НАУЧНЫЙ ХАРАКТЕР. В РАМКАХ КАНОНА ПОСТУЛИРОВАЛИСЬ ЗАКОНОМЕРНОСТЬ И НЕИЗБЕЖНОСТЬ УСПЕХОВ СОВЕТСКОГО ЗДРАВООХРАНЕНИЯ, А ИССЛЕДОВАТЕЛИ БЫЛИ ВЫНУЖДЕНЫ ЗАМАЛЧИВАТЬ НЕУДАЧИ СОВЕТСКОЙ МЕДИЦИНЫ. В ОПРЕДЕЛЕННОЙ ФОРМЕ ОН СОХРАНИЛСЯ И В ПОСТСОВЕТСКИХ ИСТОРИКО-МЕДИЦИНСКИХ УчЕьникАХ. ОБ этом См.: Sergey Zatravkin, Elena Vishlenkova, «A Ghost Textbook on the History of Medicine: A Case Study of the Legacy of a Stalinist Scholarly Canon», European Education, 52 (3), 2020, p. 257-270.

\section{AUTEUR}

\section{ВЛАДИСЛАВ ЯКОВЕНКО}

ИГИТИ ИМ. А.В. ПОЛЕТАЕВА

ФАКУЛЬТЕТ ГУМАНИТАРНЫХ НАУК, НАЦИОНАЛЬНЫЙ ИССЛЕДОВАТЕЛЬСКИЙ УНИВЕРСИТЕТ

«ВЫСШАЯ ШКОЛА ЭКОНОМИКИ»

v0395[at]yandex.ru 